\title{
Estratégias utilizadas para a prevenção do câncer de colo uterino na atenção primária em saúde: revisão da literatura
}

\author{
Moacir Andrade Ribeiro Filho ${ }^{1}$ (D), Glauberto da Silva Quirino ${ }^{2}$ (D), Mariana \\ Alexandre Gadelha de Lima ${ }^{3}$ (D) \& Maria do Socorro Lopes Rolim ${ }^{4}$ (D)
}

(1) Hospital Regional de Cajazeiras, Rua Tabelião Antonio Holanda, Cristo Rei 58900-000, Cajazeiras, Paraíba, Brasil. E-mail: moacirarf@outlook.com

(2) Universidade Regional do Cariri, Centro de Ciências da Saúde, Rua Coronel Antonio Luiz 1161, Crato 63100-000, Ceará, Brasil. E-mail: glaubertoce@hotmail.com

(3) Universidade Federal de Campina Grande, Centro de Formação de Professores, Unidade Acadêmica de Enfermagem, Rua Sérgio Moreira de Figueiredo, Casas Populares 58900-000, Cajazeiras, Paraíba, Brasil. E-mail: marianaalexandre15@gmail.com

(4) Faculdade Santa Maria, 504 BR-230, Cristo Rei 58900-000, Cajazeiras, Paraíba, Brasil. E-mail: corrinharolimm@hotmail.com

Ribeiro Filho M.A., Quirino G.S., Lima M.A.G. \& Rolim M.S.L. (2021) Estratégias utilizadas para a prevenção do câncer de colo uterino na atenção primária em saúde: revisão da literatura. Pesquisa $e$ Ensino em Ciências Exatas e da Natureza, 5(edição especial 1): e1643.

http://dx.doi.org/10.29215/pecen.v5i0.1643

Responsabilidade editorial: Maria Carmem B. de Alencar. Recebido: 03 dezembro 2020. Aceito: 13 janeiro 2021. Publicado: 03 fevereiro 2021

Resumo: No contexto da Estratégia de Saúde da Família são contempladas ações de controle do câncer de colo do útero. No entanto, essas estratégias têm se mostrado incipientes quando analisados os índices do câncer de colo uterino. $\mathrm{O}$ objetivo deste estudo é descrever a produção científica sobre as estratégias utilizadas para prevenção do câncer de colo uterino na Atenção Primária à Saúde (APS). Trata-se de uma revisão integrativa da literatura. A busca por artigos foi realizada na base de dados da Biblioteca Virtual em Saúde, SciELO, BDENF, LILACS e MEDLINE, em um recorte temporal de 2015 a 2020. A amostra foi composta por nove artigos. Verificou-se que as estratégias utilizadas foram educação popular; reuniões comunitárias; ampliação dos horários de oferta do exame; abordagem multidisciplinar com os profissionais; campanhas educativas; mutirões de coleta; busca ativa; envolvimento social; e rodas de conversas nas unidades de saúde. Estratégias são desenvolvidas, no entanto, permanece a baixa procura pelo exame, sugerindo a necessidade de reformulações e ressignificação dos processos de trabalho. Avançar no entendimento sobre a metodologia certa e ferramenta ideal para fomentar o significado que o cuidado tem para as mulheres é de fundamental importante, tendo em vista ampliar o acesso ao exame para prevenção do câncer uterino.

Palavras-chave: Neoplasias uterinas, estratégias de saúde, estratégia saúde da família, prevenção de doenças.

\section{Strategies used to prevent cervical cancer in primary health care: a literature review}

Abstract: In the context of the Family Health Strategy, actions to control cervical cancer are contemplated. However, these strategies have been shown to be incipient when analyzing cervical cancer rates. The objective of this study is to describe the scientific production on the strategies used to prevent cervical cancer in Primary Health Care (PHC). This is an integrative literature review carried out in October 2020. The search for the articles was in the database from the Virtual Health Library, 
SciELO, BDENF, LILACS and MEDLINE, in a time frame from 2015 to 2020. The sample consisted of nine articles. It was found that the strategies used were popular education; community meetings; expansion of the exam offering hours; multidisciplinary approach with professionals; educational campaigns; collection efforts; active search; social involvement; conversations in health units. Strategies are developed, however, the demand for the exam remains low, suggesting the need for reformulations and reframing of work processes. You need to advance in understanding the right methodology, the ideal tool to foste the meaning care has for women, thereby expanding access to the exam for the prevention of uterine cancer.

Key words: Uterine neoplasms, health strategies, family health strategy disease prevention.

\section{Introdução}

Todas as mulheres têm direito ao acesso à saúde integral, humanizada e de qualidade, livre de qualquer forma de preconceito ou discriminação por meio do Sistema Único de Saúde (SUS). Logo, a integralidade da assistência busca garantir ao indivíduo uma assistência à saúde que transcenda a prática curativa, contemplando o indivíduo em todos os níveis de atenção e considerando o sujeito inserido em um contexto social, familiar e cultural (Souza et al. 2012).

A saúde da mulher no contexto do câncer de colo uterino segue aquém dessa integralidade apresentada como princípio do SUS, visto que o controle e prevenção do câncer de colo uterino permanecem um desafio para os órgãos mundiais de saúde, bem como para os brasileiros, tendo em vista que, apesar de estratégias e políticas de saúde já implantadas, perduram os crescentes números de mulheres acometidas por essa neoplasia (Santos \& Varela 2015).

Como apresenta o Instituto Nacional do Câncer (INCA 2019), o câncer de colo do útero é a terceira causa de morte por câncer em mulheres no Brasil, com exceção do câncer de pele. Ele é responsável por um grande número de mortes entre as mulheres principalmente em países em desenvolvimento. Seu principal fator de risco é a infecção pelo Papiloma Vírus Humano (HPV), infecção sexualmente transmissível mais comum em todo o mundo, sendo considerada uma neoplasia de excelente prognóstico quando detectada precocemente e tratada de forma apropriada. Porém, frequentemente, o diagnóstico é tardio, ocasionando as taxas de mortalidade tão elevadas no Brasil (MSB 2013).

Atualmente, tem-se apresentado como uma das estratégias para o controle, a realização do exame preventivo do câncer de colo do útero, indicado para detecção de lesões ainda em fase inicial. O exame é ofertado pelo SUS nas Unidades Básicas de Saúde (UBS), sendo a estratégia mais adotada para detecção da doença em mulheres de 25 a 64 anos que já tiveram algum tipo de atividade sexual (INCA 2016).

Entendendo que a Atenção Primária à Saúde (APS) é uma das ferramentas capazes de desenvolver inúmeras estratégias capazes de intervirem nos altos índices de morbimortalidade por câncer de colo uterino, emergiu o seguinte questionamento: quais as estratégias utilizadas para prevenção do câncer de colo uterino na APS? Para tanto, tem-se como objetivo descrever a produção científica sobre as estratégias utilizadas na Atenção Primária em Saúde para prevenção do câncer de colo uterino.

\section{Métodos}

Esta pesquisa é uma revisão integrativa da literatura, a qual foi desenvolvida em seis etapas: (1) elaboração da pergunta norteadora; (2) busca ou amostragem na literatura; (3) coleta de dados; (4) análise crítica dos estudos incluídos; (5) discussão dos resultados; e (6) apresentação da revisão integrativa (Mendes et al. 2008).

Para ampliar a abrangência, dar rigor na formulação da pergunta problema e selecionar os descritores adequados deste estudo, utilizou-se a estratégia PVO - População/Participantes; Variáveis de interesse; Resultados/outcomes (Ursi \& Gavão 2006), conforme demonstrado na Tabela 1. 
A partir de então, a busca na literatura foi realizada durante o mês de outubro de 2020 de forma independente na Biblioteca Virtual em Saúde, Scientific Electronic Library Online (SciELO), Banco de Datos de Enfermería (BDENF), Literatura Latino-americana e do Caribe em Ciências da Saúde (LILACS) e Medical Literature Analysis and Retrieval System Online (MEDLINE), utilizando o método de busca avançada e a categoria "título, resumo e assunto".

Tabela 1. Descritores de assunto localizados no $M e S H / D e C S$ para os componentes da pergunta de pesquisa a partir da estratégia PVO.

\begin{tabular}{cclc}
\hline Acrônimo & Definição & Componentes & Descritores/MeSH \\
\hline $\mathrm{P}$ & População & $\begin{array}{l}\text { Câncer de colo uterino na Estratégia } \\
\text { Saúde da Família }\end{array}$ & $\begin{array}{c}\text { Estratégia Saúde da Família } \\
(\text { Family Health Strategy) }\end{array}$ \\
\hline $\mathrm{V}$ & Variável & $\begin{array}{l}\text { Estratégias utilizadas para prevenção } \\
\text { do câncer de colo uterino }\end{array}$ & $\begin{array}{c}\text { Estratégias de saúde } \\
(\text { Health Strategy) }\end{array}$ \\
\hline $\mathrm{O}$ & Desfecho (Outcomes) & Prevenção do câncer de colo uterino & $\begin{array}{c}\text { Prevenção de Doenças } \\
\text { (Disease Prevention) }\end{array}$ \\
\hline
\end{tabular}

Para recuperar pesquisas indexadas foram utilizados os Descritores de Ciências da Saúde (DeCS). Durante a busca realizou-se o cruzamento dos DeCS com o operador booleano AND para associação dos descritores como estratégia de busca: Family Health Strategy AND Health Strategy AND Disease Prevention.

Os cruzamentos foram realizados de forma individual em cada base, após os quais foram garimpados 174 artigos, sendo 56 na SciELO; 37 no BDENF; 54 na LILACS e 27 na MEDLINE. Em seguida, os artigos passaram por uma seleção inicial a partir da aplicação de filtros: idiomas (inglês, português e espanhol), limite temporal (escopo de 05 anos de publicação - 2015 a 2020) e textos completos disponíveis, totalizando 62 artigos filtrados. Após o processo de filtragem realizou-se a leitura dos títulos e resumos, que permitiu a exclusão de 18 estudos, restando 44 artigos. Estes foram submetidos à conferência para averiguar duplicações, identificando-se 13 duplicados, assim, resultando 31 artigos pré-selecionados.

Em seguida foram aplicados os critérios de inclusão: ser artigo original e ser referente à temática "estratégias utilizadas para prevenção de câncer de colo uterino na APS". Como critérios de exclusão: ser publicação do tipo comunicação breve, editorial, comment, artigo de reflexão, documentário, ensaio, resumo de teses, resenha, relato de experiência e projeto de intervenção; não estar disponível em texto completo para download gratuitamente, e não responder à questão de estudo.

Após a aplicação dos critérios de exclusão, os estudos foram lidos na íntegra. Nessa etapa foram excluídos 22 artigos e a coleta resultou em uma amostra final de nove artigos. Para tanto, utilizou-se o protocolo Preferred Reporting Items for Systematic Reviews and Meta-analyses (PRISMA) (Moher et al. 2015).

Seguindo orientações de Ursi \& Gavão (2006), para a extração dos dados nos artigos selecionados fez-se necessária a utilização de um instrumento, onde este permitiu assegurar que a totalidade dos dados relevantes fosse extraída.

Os resultados iniciais foram apresentados por meio da caracterização bibliométrica (Tabela 2). Em seguida, discutiu-se os achadas dessa revisão.

\section{Resultados e Discussão}

Segundo análise dos arquivos selecionados nessa revisão, o país que mais publicou sobre a temática aqui estudada foi o Brasil com oito artigos. Os anos de 2017 e 2018 foram os que mais tiveram artigos e a língua portuguesa foi a mais frequente com oito publicações (Tabela 2).

Após análise dos estudos, verificou-se que inúmeras estratégias foram desenvolvidas, sendo a maioria relacionada à educação em saúde com embasamento teórico na educação popular proposta por Paulo Freire (Correio et al. 2015; Alves et al. 2016; Silva et al. 2017). Essas estratégias utilizadas nos estudos vêm ao encontro das recomendações da Organização 


\section{Prevenção do câncer de colo uterino}

Panamericana da Saúde (OPAS 2016), que apresentam a educação em saúde e o aconselhamento como componentes essenciais de um programa efetivo de prevenção e controle do câncer do colo do útero para assegurar ampla cobertura de vacinação, alta cobertura de rastreamento e elevada adesão ao tratamento.

Tabela 2. Publicações selecionadas sobre estratégias utilizadas para prevenção do câncer de colo uterino na APS.

\begin{tabular}{|c|c|c|c|c|c|}
\hline Título do artigo & Periódico & País & Idioma & Ano & Estratégia utilizada \\
\hline $\begin{array}{l}\text { Educação popular em saúde } \\
\text { como estratégia à adesão na } \\
\text { realização do exame } \\
\text { colpocitológico }\end{array}$ & Ciência, Cuidado e Saúde & Brasil & Português & 2016 & $\begin{array}{l}\text { Educação popular - Paulo } \\
\text { Freire }\end{array}$ \\
\hline $\begin{array}{l}\text { Controle do câncer de colo } \\
\text { uterino: ações desenvolvidas } \\
\text { pelo enfermeiro à luz do } \\
\text { discurso do sujeito coletivo }\end{array}$ & $\begin{array}{l}\text { Revista Online de Pesquisa } \\
\text { Cuidado é Fundamental }\end{array}$ & Brasil & Português & 2015 & $\begin{array}{l}\text { Ações de educação em saúde; } \\
\text { convocação do público para } \\
\text { realizar o citopatológico }\end{array}$ \\
\hline $\begin{array}{l}\text { Educação em saúde como } \\
\text { estratégia de prevenção do } \\
\text { câncer do colo do útero: } \\
\text { revisão integrativa }\end{array}$ & $\begin{array}{l}\text { Revista Prevenção de Infecção } \\
\text { e Saúde - REPIS }\end{array}$ & Brasil & Português & 2017 & $\begin{array}{l}\text { Educação em Saúde: palestras } \\
\text { nas recepções }\end{array}$ \\
\hline $\begin{array}{l}\text { Intervenção na prevenção e } \\
\text { controle de câncer de colo } \\
\text { uterino e mama numa } \\
\text { unidade básica de saúde do } \\
\text { nordeste do Brasil }\end{array}$ & $\begin{array}{l}\text { Revista Brasileira de Medicina } \\
\text { de Família e Comunidade - } \\
\text { RBMFC }\end{array}$ & Brasil & Português & 2017 & $\begin{array}{l}\text { Organização e gestão, } \\
\text { monitoramento e avaliação, } \\
\text { engajamento do público e } \\
\text { qualificação da prática clínica. }\end{array}$ \\
\hline $\begin{array}{l}\text { Construção de um material } \\
\text { educativo para a prevenção } \\
\text { do câncer de colo do útero }\end{array}$ & $\begin{array}{l}\text { Estudos Interdisciplinares em } \\
\text { Psicologia }\end{array}$ & Brasil & Português & 2017 & $\begin{array}{l}\text { Confecção de uma cartilha } \\
\text { em formato de flor }\end{array}$ \\
\hline $\begin{array}{l}\text { The impact of a human } \\
\text { papillomavirus (HPV) } \\
\text { vaccination campaign on } \\
\text { routine primary health } \\
\text { service provision and health } \\
\text { workers in Tanzania: a } \\
\text { controlled before and after } \\
\text { study }\end{array}$ & BioMed Central & Tanzânia & Inglês & 2018 & Vacinação do HPV \\
\hline $\begin{array}{l}\text { Prevenção de câncer de colo } \\
\text { uterino e de mama num } \\
\text { município do sul do país. }\end{array}$ & $\begin{array}{l}\text { Arquivos Catarinenses de } \\
\text { Medicina - ACM }\end{array}$ & Brasil & Português & 2018 & $\begin{array}{llr}\text { Implementação } & \text { de } & \text { um } \\
\text { projeto de intervenção } & \text { com } \\
\text { ampliação do horário } & \text { de } \\
\text { coleta } & & \\
\end{array}$ \\
\hline $\begin{array}{l}\text { A organização do } \\
\text { rastreamento do câncer do } \\
\text { colo uterino por uma equipe } \\
\text { de Saúde da Família no Rio } \\
\text { de Janeiro, Brasil. }\end{array}$ & $\begin{array}{l}\text { Revista Brasileira de Medicina } \\
\text { de Família e Comunidade - } \\
\text { RBMFC }\end{array}$ & Brasil & Português & 2018 & $\begin{array}{l}\text { Busca ativa de mulheres que } \\
\text { não frequentam a unidade, } \\
\text { com agendamento imediato } \\
\text { de coleta, oferta racional de } \\
\text { coleta facilitada para } \\
\text { mulheres na unidade de } \\
\text { saúde }\end{array}$ \\
\hline $\begin{array}{l}\text { Ações educativas: papel } \\
\text { da(o) enfermeira(o) na } \\
\text { prevenção do câncer do } \\
\text { colo do útero }\end{array}$ & $\begin{array}{l}\text { Brazilian Journal } \\
\text { Development - BJD }\end{array}$ & Brasil & Português & 2019 & $\begin{array}{l}\text { Ações Educativas: nas visitas } \\
\text { domiciliares }\end{array}$ \\
\hline
\end{tabular}

Corroborando Falkenberg et al. (2014) defendem que educação em saúde deve ser enfatizada nas ações estratégicas em saúde, fortalecendo a educação popular em saúde, que valoriza os saberes, o conhecimento prévio da população e não somente o conhecimento científico. Para esses autores, deve ser lançada mão também da educação permanente em saúde, de maneira a buscar as lacunas de conhecimento dos profissionais, ações direcionadas a qualificação dos processos de trabalho em saúde considerando as especificidades locais e as necessidades do trabalho real.

A educação em saúde tem sido essencial no campo da APS. Ela é transversal nos diversos eixos temáticos que envolvem o emponderamento dos usuários, cuidadores e profissionais de saúde, estimulando-os a agir conscientemente diante de cada ação do cotidiano, criando um espaço para o aprimoramento de novos conhecimentos e práticas. 
O Ministério da Saúde do Brasil (MSB 2006) define educação em saúde como um "processo educativo de construção de conhecimentos em saúde que visa à apropriação temática pela população [...]. Conjunto de práticas do setor que contribui para aumentar a autonomia das pessoas no seu cuidado e no debate com os profissionais e os gestores a fim de alcançar uma atenção à saúde de acordo com suas necessidades”.

Um trabalho realizado por Alves \& Aertes (2011) mostrou que outras formas de educação em saúde eram caracterizadas por ações verticais de caráter informativo com o intuito de transformar hábitos de vida, colocando o indivíduo como o responsável pela sua saúde. Por meio dessa compreensão, a ação de prevenir envolve também a questão educativa de disseminar conhecimento para que as próprias pacientes se conscientizem e percebam a importância do exame como instrumento diagnóstico (Cunha 2016).

Nesse contexto, para Colomé \& Oliveira (2012), o sucesso da prevenção do câncer de colo uterino relaciona-se a programas educativos de diversas naturezas e à valorização da cultura estritamente relacionada ao conhecimento da doença e das formas de realizar sua prevenção. $\mathrm{O}$ desenvolvimento da autonomia e o enfrentamento de novas situações, propiciados pela interação no grupo, permitem aos usuários maior controle de seu contexto social e ambiental. Durante o processo de educação em saúde é fundamental que se considere o indivíduo como detentor de conhecimento e não mero receptor de informações.

O Controle do Câncer Uterino (CCU) no setor público condiz com ações de gestão e dos profissionais de saúde, organizadas segundo os níveis hierárquicos do SUS, de modo articulado, compondo uma atenção à saúde na perspectiva de integralidade, sendo que essas ações são norteadas por uma linha de cuidado que sinaliza o fluxo assistencial e os correspondentes protocolos e diretrizes clínicas diante aos graus de evolução da enfermidade (INCA 2012).

Isso corrobora com o estudo de Romero et al. (2017), quando eles apresentaram um relato de experiência baseada em quatro eixos, que consiste em ações de organização e gestão do serviço, engajamento público, qualificação da prática clínica e monitoramento e avaliação com o foco na prevenção e controle do câncer de colo de útero e de mama.

Essa integração, gestão, comunidade e serviço tem contribuído para a organização dos serviços de prevenção, consequentemente, garantido um acesso de qualidade e integrado entre a rede de atenção à saúde, logo, à extensão de serviços, e à mobilização da comunidade. As mensagens de educação em saúde sobre o câncer do colo do útero devem refletir a política nacional e ser culturalmente apropriadas e uniformes em todos os níveis do sistema de saúde.

A educação em saúde deve estar inserida no cotidiano da Estratégia de Saúde da Família, visto que, as práticas de educação em saúde são inerentes ao trabalho em saúde, mas muitas vezes estão relegadas a um segundo plano no planejamento e organização dos serviços, na execução das ações de cuidado e na própria gestão.

Perpassando por essa concepção, trabalhar a prevenção de doenças com base no contexto da educação em saúde, faz-se necessário através da busca de um diálogo sobre modos de vida e emponderamento social e individual de uma pessoa. Com isso, surge o desafio de mudar o paradigma de vida de uma pessoa que foi construído sob uma base conceitual de que saúde é ausência de doenças, onde sua saúde depende apenas dos serviços de saúde e não de sua participação na construção dessa autonomia, ou seja, mudança de comportamento.

Sob a perspectiva de buscar a saúde apenas quando se está doente, o estudo de Silva et al. (2017) revelou que $50 \%$ das mulheres somente comparecem à consulta médica quando apresentam algum agravo a sua saúde, pois têm o entendimento de que procurar a UBS é sinal de estar doente.

Somando-se a tudo isto, existe a realidade das infraestruturas das instituições, em particular as públicas, que associada à postura dos profissionais de saúde e a ausência de uma política de saúde efetiva, interferem na adesão ao exame Papanicolau, na análise da cobertura do exame preventivo e na sua baixa demanda.

Ressalta-se que o fato de simplesmente ofertar o exame preventivo não é suficiente para que as mulheres estejam prevenidas desta doença. Faz-se necessária uma intensa mobilização das 
mulheres através da educação em saúde e a participação profissional no desenvolvimento de suas responsabilidades para com os serviços de prevenção de câncer ginecológico.

O MSB (2013) apresenta as atribuições a desenvolver no nível da atenção primária, que são da responsabilidade sanitária da equipe, e incluem: esclarecer e informar a população feminina sobre o rastreamento, identificar na área aquelas que pertencem à faixa etária prioritária e grupos de risco, convocar e realizar a coleta de citologia, e detectar e reconvocar as que se ausentaram. E ainda, o recebimento dos laudos, captação dos resultados positivos para vigilância do caso, orientação e encaminhamento à atenção secundária, avaliação da cobertura de citologia na área e qualidade da coleta.

Outras estratégias utilizadas foram apresentadas por Zinhani et al. (2018); ampliação dos horários de oferta do exame; promoção de uma abordagem multidisciplinar com os profissionais (educação em saúde); campanhas educativas; mutirões de coleta; visitas domiciliares (Ferraz et al. 2019). Eles mostraram que essas estratégias conseguiram ampliar o acesso de mulheres à realização da coleta citopatológica de colo uterino. Além disso, foi observado com essa ampliação que outros grupos de mulheres realizaram o exame, dentre essas, as que trabalhavam nos horários em que as UBS estavam abertas e as que só tinham o horário do almoço para a realização do exame.

Percebe-se que estratégias apresentadas anteriormente são comuns à maioria dos estudos, somando-se à construção de material educativo (Peuker et al. 2017). Essas ferramentas de promoção da saúde não divergem das apresentadas por outros estudos, inclusive, internacionais, que defendem a efetividade dessas estratégias quando são planejadas e executadas sob a perspectiva da coletividade.

A experiência do Reino Unido aponta que a utilização de cartas-convites à cobertura do exame Papanicolau aumentou para $85 \%$ da adesão à coleta, logo a incidência da doença reduziu cerca de $50 \%$. Assim, houve a mudança do rastreamento oportunístico, que era realizado num momento de um atendimento eventual, para um rastreamento organizado (MSB 2013).

Segundo Corrêa et al. (2012), no Brasil, o rastreamento cervical ocorre, na sua maioria, de maneira oportunística, ou seja, existe um dia específico para essa coleta, e apenas as mulheres que procuram o serviço realizam o exame, não havendo nenhum esforço para o direcionamento à população de maior risco ou para as que nunca realizaram a coleta citopatológica. A consequência disso é a repetição frequente dos exames, caracterizando falta de controle sob quem são as mulheres do grupo de risco e as que nunca realizam a coleta citológica do colo uterino.

Quando se trata de conhecimento sobre o exame e de sua importância para melhorar a qualidade de vida da população feminina, os profissionais ainda encontram barreiras que se estendem da baixa escolaridade ao preconceito no que tange a realização do exame. Neste contexto, é válido lembrar que em momentos oportunos e estratégicos, as equipes de atenção básica têm desenvolvido um papel primordial no tocante à educação em saúde, esclarecendo ao público feminino detalhes sobre o exame e sua importância para reduzir a incidência de câncer (Maia et al. 2018).

Essas constatações apontam para a necessidade de um planejamento participativo e de adoção de uma estratégia capaz de envolver todos os atores no processo de educação em saúde. É fato que essas barreiras intelectuais, conceituais e culturais precisam ser derrubadas, sendo um dos principais desafios para os profissionais na atualidade, pois, promover o empoderamento feminino para adesão ao exame através da educação em saúde permanece um gargalo.

Tiensoli et al. (2018) concordam, para eles, quando se trata da adesão das mulheres à realização do exame colpocitopatológico, percebe-se que a baixa escolaridade é um determinante social para o desenvolvimento do câncer de colo do útero. A escolaridade constitui um importante mediador da relação entre nível socioeconômico e percepção da saúde, considerando que indivíduos com maiores níveis de escolaridade adotam estilos de vida mais saudáveis, provavelmente devido ao acesso facilitado aos serviços avançados de saúde e ao maior conhecimento sobre a doença e suas formas de prevenção. 
Segundo Corrêa \& Russomano (2012), o sucesso histórico do modelo convencional de prevenção, baseado no rastreio citológico de lesões precursoras, na redução da incidência e da mortalidade por câncer do colo do útero e inegável. Todavia, atualmente, essa estratégia de prevenção está sofrendo importantes questionamentos, sendo objeto de estudos que buscam aperfeiçoá-la ou confrontá-la com novas alternativas.

As tendências atuais na área da educação em saúde apontam para a utilização de metodologias ativas de ensino-aprendizagem, visando tornar o cliente o protagonista do seu próprio processo de autocuidado. As metodologias ativas também vão ao encontro da pedagogia da autonomia, a qual preconiza a capacidade de se autogerenciar ou autogovernar o seu próprio cuidado (Pedrosa et al. 2011).

A vacinação contra o HPV foi também identificada como uma estratégia utilizada para prevenir o câncer uterino (Gallagher et al. 2018), eles realizaram uma campanha de vacinação nas escolas da Tanzânia defendendo a máxima que a oferta de vacina contra o HPV remetem uma redução futura na incidência do HPV principal causador do câncer de colo uterino. Corroborando com esse estudo, Borsatto et al. (2011) mostram que as vacinas contra o HPV podem ser profiláticas, limitando a infecção pelo vírus e as doenças dele decorrentes, sendo considerado um instrumento de prevenção primária ou terapêutica, quando induzem a regressão de lesões precursoras e a remissão do câncer.

A OPAS (2016) recomenda que as vacinas contra HPV devam ser introduzidas como parte de uma estratégia coordenada para a prevenção do câncer colo do útero e de outras doenças relacionadas ao HPV e, principalmente, não deve diminuir ou desviar recursos dos programas de rastreamento, pois sua continuidade é imprescindível.

A prevenção pode ser realizada por meio de vacina e da adoção de práticas sexuais seguras, como o uso de preservativos nas relações sexuais. Porém, há algumas limitações que devem ser reconhecidas: a vacina não inclui todos os subtipos oncogênicos do HPV, e a transmissão do HPV pode ocorrer também a partir do contato pele a pele das áreas genitais próximas ao pênis e à vagina (Tiensoli 2018).

Conforme orientado pelo INCA (2019), são necessárias ações de comunicação, planejamento, monitoramento e avaliação para o sucesso dessa estratégia. As etapas do rastreio implicam desde a identificação e convite às mulheres, garantia dos recursos humanos e materiais, disponibilização de exames de qualidade, até o seguimento das mulheres assegurando tratamento e cuidados para aquelas com exames alterados.

As estratégias de promoção em saúde devem ser elaboradas com o intuito de melhorar a qualidade do cuidado, a adequação do atendimento, o custo-efetividade e para servir como ferramentas educacionais. Logo, essas diretrizes devem ser vistas como uma importante estratégia de transformação social, devendo estar vinculada às lutas sociais mais simples e ser assumida pela equipe de saúde, reorientando as práticas de saúde e as relações que se estabelecem entre o cotidiano e o saber da saúde.

\section{Considerações finais}

Tomando por base as constatações identificadas nos estudos garimpados, denota-se que inúmeras estratégias de educação em saúde são desenvolvidas no campo da APS. No entanto, os dados estatísticos referentes ao controle do câncer de colo uterino apontam para a necessidade de ações e estratégias capazes de reverter à realidade dos persistentes índices desse tipo de câncer ginecológico.

Dentre essas, sugere-se avançar no entendimento sobre a metodologia certa, a ferramenta ideal para fomentar o significado que o cuidado tem para as mulheres, com isso ampliar o acesso aos serviços ofertados, intervindo no modo de operar do cotidiano profissional, logo, buscar a reformulações e ressignificação dos processos de trabalho no campo da APS.

No contexto da Estratégia Saúde da Família são contempladas algumas ações por parte dos profissionais em relação ao controle do câncer de colo do útero. Não obstante, essas estratégias realizadas na internalidade de cada unidade de saúde têm se mostrado incipientes por 
serem planejadas em espaços fechados e sem a contribuição das mulheres participantes dessa desse processo.

É importante, então, a utilização de metodologias de ensino-aprendizagem participativas e dialógicas, tais como as utilizadas nas ações de educação popular em saúde, inseridas nos currículos de educação continuada e nas ações de educação permanente em saúde, visando uma formação profissional em saúde mais adequada às necessidades de saúde individuais e coletivas, na perspectiva da equidade e da integralidade.

As conclusões dessa revisão são preliminares e não podem ser consideradas definitivas, devido aos vieses possíveis nos estudos primários, como o recorte temporal, limite da busca, caracterizando limitação na pesquisa.

Assim, persiste o entendimento de que, para uma estratégia de prevenção, faz-se necessário o envolvimento da gestão, ensino, comunidade, serviços, bem como uma política pública de saúde efetiva e uma ressignificação nos modus operandi de cada estratégia a ser utilizada. Ademais, sugerem-se aprofundamentos teóricos metodológicos relacionados à promoção de saúde, capaz de preencher as lacunas encontradas nesse estudo, sobretudo, fortalecer a integralidade da assistência na perspectiva do controle de câncer de colo uterino.

\section{Referências}

Alves G.G. \& Aerts D. (2011) As práticas educativas em saúde e a Estratégia Saúde da Família. Ciência \& Saúde Coletiva, 6(1): 319-325. https://doi.org/10.1590/S1413-81232011000100034

Alves S.R., Alves A.O. \& Assis M.C.S. (2016) Educação popular em saúde como estratégia à adesão na realização do exame colpocitológico. Ciência, Cuidado e Saúde, 15(3): 570-574. https://doi.org/10.4025/cienccuidsaude.v15i3.27125

Borsatto A.Z., Vidal M.L.B. \& Rocha R.C.N.P. (2011) Vacina contra o HPV e a Prevenção do Câncer do Colo do Útero: Subsídios para a Prática. Revista Brasileira de Cancerologia, 57(1): $67-74$.

Colomé J.S. \& Oliveira D.L.L.C. (2012) Educação em saúde: por quem e para quem? A visão de estudantes de graduação em enfermagem. Texto \& Contexto-Enfermagem, 21(1): 177-184. https://doi.org/10.1590/S0104-07072012000100020

Corrêa F.M. \& Russomano F.B. (2012) Novas Tecnologias de Prevenção do Câncer do Colo do Útero: Quem de Fato se Beneficia? Revista Brasileira de Cancerologia, 58(3): 525-527. https://doi.org/10.32635/2176-9745.RBC.2012v58n3.1411

Corrêa D.A.D., Villela W.V. \& Almeida A.M. (2012) Desafios à organização de Programa de rastreamento do câncer do colo do útero em Manaus-AM. Texto \& Contexto-Enfermagem, 21(2): 395-400. https://doi.org/10.1590/S0104-07072012000200018

Correio K.D.L., Ramos A.I.G., Santos R.L.G., Bushatsky M. \& Correio M.B.S.C.B. (2015) Controle do câncer do colo do útero: ações desenvolvidas pelo enfermeiro à luz do discurso do sujeito coletivo. Revista Online de Pesquisa Cuidado é Fundamental, 7(2): 2425-2439. https://doi.org/10.9789/2175-5361.2015.v7i2.2425-2439

Cunha F.A. (2016) Atenção primária à saúde da mulher: enfoque educativo preventivo no combate ao câncer de colo uterino em pacientes assistidas pela unidade de saúde de soledade II-Natal. Monografia (Especialização em Atenção Básica em Saúde). Programa Mais Médicos. Universidade Federal do Maranhão, São Luís, Maranhão.

Falkenberg M.B., Mendes T.P.L., Moraes E.P. \& Souza E.M. (2014) Educação em saúde e educação na saúde: conceitos e implicações para a saúde coletiva. Ciência \& Saúde Coletiva, 19(3): 847852. https://doi.org/10.1590/1413-81232014193.01572013

Ferraz E.T.R., Jesus M.E.F. \& Leite R.N.Q. (2019) Ações educativas: papel da (o) enfermeira (o) na prevenção do câncer do colo do útero. Brazilian Journal of Development., 5(10): 21083-21093. https://doi.org/10.34117/bjdv5n10-271

Gallagher K.E., Erio T., Baisley K., Lees S. \& Watson-Jones D. (2018) The impact of a human papillomavirus (HPV) vaccination campaign on routine primary health service provision and 
health workers in Tanzania: a controlled before and after study. BMC Health Services Research, 18(173): 1-10. https://doi.org/10.1186/s12913-018-2976-2

INCA (2012) Ministério da Saúde. Instituto Nacional de Câncer José Alencar Gomes da Silva. ABC do câncer: abordagens básicas para o controle do câncer. $2^{\circ}$ edição. Rio de Janeiro: INCA. $32 \mathrm{p}$.

INCA (2016) Ministério da Saúde. Instituto Nacional de Câncer José Alencar Gomes da Silva. Coordenação Geral de Ações Estratégicas. Divisão de apoio à Rede de Atenção Oncológica. Diretrizes Brasileiras para o rastreamento do câncer do colo do útero. $2^{\circ}$ edição. Rio de Janeiro: INCA. 114 p.

INCA (2019) Ministério da Saúde. Instituto Nacional de Câncer José Alencar Gomes da Silva. Parâmetros técnicos para o rastreamento do câncer do colo do útero. Rio de Janeiro: INCA. 32 p.

Maia M.N., Silva R.P.O. \& Santos L.P.R. (2018) A organização do rastreamento do câncer do colo uterino por uma equipe de Saúde da Família no Rio de Janeiro, Brasil. Revista Brasileira de Medicina de Família e Comunidade, 13(40): 1-10. https://doi.org/10.5712/rbmfc13(40)1633

Mendes K.D.S., Silveira R.C.C.P. \& Galvão C.M. (2008) Revisão integrativa: método de pesquisa para a incorporação de evidências na saúde e na enfermagem. Texto \& ContextoEnfermagem, 17(4): 758-764. https://doi.org/10.1590/S0104-07072008000400018

Moher D., Liberati A., Tetzlaff J. \& Altman D.G. (2015) Principais itens para relatar Revisões sistemáticas e Meta-análises: A recomendação PRISMA. Epidemiologia Serviços Saúde, 24(2):335-342. http://dx.doi.org/10.5123/S1679-49742015000200017

MSB (2006) Ministério da Saúde do Brasil. Secretaria-Executiva. Secretaria de Gestão do Trabalho e da Educação na Saúde. Glossário temático: gestão do trabalho e da educação da saúde. $2^{\circ}$ edição. Brasília: Ministério da Saúde. 44 p.

MSB (2013) Ministério da Saúde do Brasil. Secretaria de Atenção à Saúde. Departamento de Atenção Básica. Controle dos cânceres do colo do útero e da mama. $2^{\circ}$ edição. Brasília: Ministério da Saúde. 124 p.

OPAS (2016) Organização Pan-Americana da Saúde. Controle integral do câncer do colo do útero. Guia de práticas essenciais. Washington: OPAS. 415 p.

Pedrosa I.L., Lira G.A., Oliveira B., Silva M.S.M.L., Santos M.B., Silva E.A. \& Freire D.M.C. (2011) Uso de metodologias ativas na formação técnica do agente comunitário de saúde. Trabalho Educação e Saúde, 9(2): 319-332. https://doi.org/10.1590/S1981-77462011000200009

Peuker A.C., Lima N.B., Freire K.M., Oliveira C.M.M. \& Castro E.K. (2017) Construção de um material educativo para a prevenção do câncer de colo do útero. Estudos Interdisciplinares em Psicologia, 8(2): 146-160. https://doi.org/10.5433/2236-6407.2017v8n2p146

Romero L.S., Shimocomaqui G.B. \& Medeiros A.B.R. (2017) Intervenção na prevenção e controle de câncer de colo uterino e mama numa unidade básica de saúde do nordeste do Brasil. Revista Brasileira de Medicina de Família e Comunidade, 12(39): 1-9. https://doi.org/10.5712/rbmfc12(39)1356

Santos A.C.S. \& Varela C.D.S. (2015) Prevenção do câncer de colo uterino: motivos que influenciam a não realização do exame de Papanicolaou. Revista Enfermagem Contemporânea, 4(2): 179-188.

Silva L.R., Almeida C.A.P.L., Sá G.G.M., Moura L.K.B \& Araújo E.T.H. (2017) Educação em saúde como estratégia de prevenção do câncer do colo do útero: revisão integrativa. Revista Prevenção de Infecção e Saúde, 3(4): 35-45. https://doi.org/10.26694/repis.v3i4.6708

Souza M.C., Araújo T.M., Júnior W.M.R., Souza J.N., Vilela A.B.A. \& Franco T.B. (2012) Integralidade na atenção à saúde: um olhar da Equipe de Saúde da Família sobre a fisioterapia. O Mundo da Saúde, 36(3): 452-460.

Tiensoli S.D., Felisbino-Mendes M.S. \& Velasquez-Melendez G. (2018) Avaliação da não realização do exame Papanicolaou por meio do Sistema de Vigilância por inquérito telefônico. Revista Escola de Enfermagem da USP, 52(e03390): 1-7.

https://doi.org/10.1590/s1980-220x2017029503390 
Ursi E.S. \& Gavão C.M. (2006) Prevenção de lesões de pele no perioperatório: revisão integrativa da literatura. Revista Latino-Americana de Enfermagem, 14(1): 124-131. https://doi.org/10.1590/S0104-11692006000100017

Zinhani M.C., Cortes H.M., Casarin S.T. \& Budel F. (2018) Prevenção de câncer de colo uterino e de mama num município do Sul do país. Arquivos Catarinenses de Medicina, 47(2): 23-34. 